\title{
Dynamic pore collapse in viscoplastic materials
}

\author{
W. Tong and G. Ravichandran \\ Division of Engineering and Applied Science, California Institute of Technology, Pasadena, \\ California 91125
}

(Received 23 January 1993; accepted for publication 29 April 1993)

\begin{abstract}
Dynamic pore collapse in porous materials is studied by analyzing the finite deformation of an elastic/viscoplastic spherical shell under impulsive pressure loading. Effects of dynamic loading rate, pore size, initial porosity, strain-rate sensitivity, strain hardening, thermal softening, and mass density of the matrix material on the pore collapse process are examined and results are compared with those from quasistatic analyses of both rate-independent and rate-dependent matrix materials. Dynamic (incrtia) effects are found to be significant or even dominant in certain shock wave consolidation conditions. An approximate method is proposed to incorporate dynamic effects into quasistatic pore-collapse relations of viscoplastic matrix materials. Implications of results of current study are discussed in terms of understanding the processes of shock wave consolidation of powders.
\end{abstract}

\section{INTRODUCTION}

Dynamic consolidation of metallic and ceramic powders provides an alternate approach for processing materials with unique microstructures and, hence, properties. ${ }^{1-6}$ This process holds to be a promising new technology for consolidating advanced materials such as nanophase intermetallics and composites. However, several major difficulties hinder its practical application and remain to be overcome, such as criteria for optimization of processing parameters (shock pressure, shock duration, etc.) and the cracking of compacts that occurs during shock release to ambient pressure. Researchers have continued to improve dynamic compaction fixtures, the use of clcancr powders, postconsolidation annealing or isostatic hot pressing, and other means to address these problems. However, the determination of shock consolidation processing parameters and prevention of cracking depends upon the detailed understanding of the dynamic consolidation process at both continuum and microstructural levels.

The densification and bonding of particles are accomplished by the passage of a strong shock wave through an initially porous material. During the densification most of the energy is deposited near the particle surfaces and the resulting heating produces melting of the particle surfaces that solidify rapidly via heat conduction into the interior of the particles before release of shock pressure. ${ }^{2}$ The physical phenomena of such a dynamic consolidation process at the particle level are very complex and remain poorly understood. Localized energy depositions at interparticle surfaces are thought to be due to local plastic deformation and frictional sliding and contribute largely to the particleparticle bonding. Berry and Williamson ${ }^{7}$ presented a computer simulation of the shock wave compaction of stainless-steel cylinders by considering plastic deformation at the particle level and predicted indeed highly localized plastic deformation and large temperature rises occur at particle boundaries during consolidation. Their numerical results have been qualitatively confirmed by experimental observations. ${ }^{8,9}$ Several estimates of the shock wave pressure required for obtaining good compact of metallic pow- ders (fully densified with good mechanical strength) have been proposed by Schwarz et al. $^{2}$ and Gourdin ${ }^{3}$ using an energy partition and balance approach. Ferreira and Meyers ${ }^{10}$ also used a similar energy balance approach by including the effect of the matrix material strength on the required compaction pressure for various materials. The lower bound on the shock duration is also given by Schwarz et al. ${ }^{2}$ by requiring the duration of shock compaction pulse to exceed the time for the melt to solidify. In all of these analyses, the total energy input during shock consolidation is determined through the equation of state of porous materials, such as the one first described by Herrmann ${ }^{11}$ and modified by Carroll and Holt ${ }^{12}$ and Swegle. ${ }^{13}$ In these porous material models, the volume change due to pore collapse is separated from that due to volume change of the matrix material. It is assumed that the form of the function that relates pressure to specific volume and specific internal energy of porous material is the same as that of the fully densified matrix material. To complete the model, a pore-collapse relation that relates pressure $p$ to the distention of the porous material $\alpha$ is needed and has generally been assumed to be an algebraic relation between pressure and distention, $\alpha=g(p)$. The matrix material is essentially treated to be elastic/perfectly plastic, rate independent, and dynamic effects are neglected. This type of equation of state of porous materials has also been commonly used in computer simulation of shock consolidation experiments. ${ }^{8}$

Both high-pressure loading rates and high rates of deformation are encountered in the consolidation process of powders by the passage of a shock wave. Shock pressures of 1-10 GPa or higher are commonly used and typical shock rise times are of the order of $100-1000 \mathrm{~ns} ;{ }^{14,15}$ thus, the typical pressure rate is $1-100 \mathrm{MPa} / \mathrm{ns}$ or higher. The initial relative density of powders used in shock wave consolidation is usually around $0.5-0.75$ and hence the inelastic volumetric strain is about $0.3-0.7$ after consolidation. The overall strain rate can be estimated to be of the order of $10^{5}-10^{7}$ per second within the shock wave front (dividing the volumetric strain by the shock wave rise time). 
Carroll and Holt ${ }^{16}$ showed that there is a significant difference between the static and dynamic pore-collapse relations for perfectly plastic and rate-independent matrix materials under these pressure rates of shock wave loading. It should be also noted that the matrix material model used in the dynamic analyses by both Berry and Williamson ${ }^{7}$ and Carroll and Holt ${ }^{16}$ is perfectly plastic and rate independent. There is increasing experimental evidence that indicates that some metals become strongly rate dependent at very high strain rates. ${ }^{17-19}$ While there have been some empirical and analytical formulations on the densification process of viscoplastic porous materials under quasistatic conditions, ${ }^{20-25}$ there are relatively few on the dynamic pore collapse of such matrix materials. ${ }^{8,26}$ The inertial effect combined with strain rate, strain-hardening, and thermal softening effects on dynamic consolidation of powders remains unclear and thus have not been included in the determination of consolidation process parameters. The applicability of relatively simple results of viscoplastic porous matrix materials under quasistatic conditions to dynamic loading conditions is also not known.

Analogous to the work by Carroll and Holt ${ }^{16}$ on the dynamic pore collapse of perfectly plastic, rateindependent materials, here the collapse of an elastic/ viscoplastic spherical shell under an external impulsive pressure loading is studied. The thermal softening effect is also included by considering adiabatic heating of the matrix material due to accumulated plastic work. The collapse of a spherical shell is an idealization of the consolidation process and does not consider other effects such as the shape and distribution of pores, but it does provide a basis in terms of simple analytical and/or numerical solutions which can be readily obtained, and the effect of dynamic loading, rate-dependent, and temperaturedependent behavior of the matrix material on pore-collapse process can be directly assessed. Our results indicate that parameters characterizing strain rate sensitivity, strain hardening, thermal softening, mass density of matrix material, dynamic loading rate, pore size, and initial relative density of the porous material play a significant role on the pore collapse under impulsive pressure loading, such as the characteristic time of densification. For viscoplastic matrix materials, rate sensitivity, dynamic effects, and others should be assessed carefully in formulating a realistic $p-\alpha$ relation for a given material under a particular loading condition. For rate-dependent matrix materials, the densification process is significantly slowed down at high loading rates.

In the following section a complete formulation of finite elastic/viscoplastic deformation of a spherical shell is presented. In Sec. III results of a series of numerical sinulations of dynamic pore collapse process are presented. Finally, discussions and conclusions of the present study are given in Sec. IV.

\section{FINITE DEFORMATION FORMULATION}

Following the approach used by Carroll and $\mathrm{Holt}^{16}$ and Wilkinson and Ashby, ${ }^{20}$ we study the consolidation of porous materials by considering the collapse of a spherical shell under a given external pressure loading $p(t)$. The current inner and outer radii $r$ of the spherical shell are $a$ and $b$, respectively, at the time $t>0$. The initial inner and outer radii $R$ are $A$ and $B$, respectively (i.e., $t=0$ ). The initial $f_{0}$ and current $f$ volume fractions of porosity in the material are thus given by

$f_{0}=(A / B)^{3}, \quad f=(a / b)^{3}, \quad 0<f_{0}<1,0<f \leqslant 1$,

the initial $\alpha_{0}$ and current $\alpha$ distentions of the porous material are given by

$\alpha_{0}=\frac{1}{1-(A / B)^{3}}, \quad \alpha=\frac{1}{1-(a / b)^{3}}, \quad 1<\alpha_{0}, \quad 1<\alpha$,

and the initial $\bar{\rho}_{0}$ and current $\bar{\rho}$ relative densities are given by

$$
\bar{\rho}_{0}=1-(A / B)^{3}, \quad \bar{\rho}=1-(a / b)^{3}, \quad 0<\bar{\rho}_{0}<1, \quad 0<\bar{\rho}<1 .
$$

Because we are interested in the whole pore-collapse process as the final relative density of the porous material approaching unity, it warrants a finite deformation analysis, In this section we outline the finite deformation formulation for stress wave propagation in elastic/viscoplastic materials applied to a spherical shell. The elastic deformation and an appropriate constitutive model are also included in our analysis. Tong and co-workers ${ }^{19}$ have presented the corresponding finite deformation formulation for elastic/viscoplastic materials in plane strain.

\section{A. Kinematics}

For a spherical shell, let $\mathbf{x}$ represent a material element in the current configuration (i.e., the spherical coordinates at time $t>0$ ) that was initially at $\mathbf{X}$ in the reference configuration (i.e., the spherical coordinates at time $t=0$ ). The deformation of the spherical shell under a pressure loading can be represented by

$$
\mathbf{x}=\hat{\mathbf{x}}(\mathbf{X}, t)=r(R, t) \mathbf{e}_{R},
$$

where $\mathrm{X}=R \mathrm{e}_{R}, R$ is the radial coordinate in the reference configuration $(A \leqslant R<B), r$ is the radial coordinate in the current configuration $[a(t) \leqslant r(R, t) \leqslant b(t)], \mathrm{e}_{R}$ is the unit basis vector in the radial direction.

The deformation gradient $\mathbf{F}$ is then given by ${ }^{27}$

$$
\mathbf{F}=\frac{\partial \mathbf{x}}{\partial \mathbf{X}}=\left[\begin{array}{ccc}
\frac{\partial r}{\partial R} & 0 & 0 \\
0 & \frac{r}{R} & 0 \\
0 & 0 & \frac{r}{R}
\end{array}\right]=\left[\begin{array}{ccc}
\frac{\eta R^{2}}{r^{2}} & 0 & 0 \\
0 & \frac{r}{R} & 0 \\
0 & 0 & \frac{r}{R}
\end{array}\right]
$$

Here $\eta$ is the ratio of the current volume to the reference volume of the matrix material, i.e., $\operatorname{det} \mathbf{F}=\eta(R, t)$.

The rate of change of the deformation gradient $F$ is 


$$
\dot{\mathbf{F}}=\left[\begin{array}{ccc}
\frac{\partial}{\partial t}\left(\frac{\partial r}{\partial R}\right) & 0 & 0 \\
0 & \frac{1}{R} \frac{\partial r}{\partial t} & 0 \\
0 & 0 & \frac{1}{R} \frac{\partial r}{\partial t}
\end{array}\right] \quad \quad \overrightarrow{\mathbf{T}}=\mathbf{F T}^{T}=\left[\begin{array}{ccc}
\frac{\eta R^{2}}{r^{2}} T_{R} & 0 & 0 \\
0 & \frac{r}{R} T_{\theta} & 0 \\
0 & 0 & \frac{r}{R} T_{\theta}
\end{array}\right]
$$

$$
=\left[\begin{array}{ccc}
\frac{\dot{\eta} R^{2}}{r^{2}}-\frac{2 \eta R^{2} u}{r^{3}} & 0 & 0 \\
0 & \frac{u}{R} & 0 \\
0 & 0 & \frac{u}{R}
\end{array}\right]=\left[\begin{array}{ccc}
\frac{\partial u}{\partial R} & 0 & 0 \\
0 & \frac{u}{R} & 0 \\
0 & 0 & \frac{u}{R}
\end{array}\right] \text {, }
$$

where the radial particle velocity $u$ is defined as $u(R, t)$ $=\partial r(R, t) / \partial t$. The corresponding spatial velocity gradient is

$$
\mathbf{L}=\mathbf{D}+\mathbf{W}=\dot{\mathbf{F}} \mathbf{F}^{-1}=\left[\begin{array}{ccc}
\frac{r^{2}}{\eta R^{2}} \frac{\partial u}{\partial R} & 0 & 0 \\
0 & \frac{u}{r} & 0 \\
0 & 0 & \frac{u}{r}
\end{array}\right],
$$

where $\mathbf{D}=\left(\mathbf{L}+\mathbf{L}^{T}\right) / 2$ is the rate of deformation tensor and $\mathbf{W}=\left(\mathbf{L}-\mathbf{L}^{T}\right) / 2$ is the spin rate tensor. However, for the axisymmetric deformations considered here, $W=0$ and thus $\mathbf{D}=\mathbf{L}$.

\section{B. Momentum balance and constitutive relation}

The balance of linear momentum in a spherical coordinates is given ss $^{27}$

$$
\frac{\partial T_{R}}{\partial R}+\frac{2}{R}\left(T_{R}-T_{\theta}\right)=\rho_{0} \frac{\partial u}{\partial t},
$$

where $T_{R}$ and $T_{\theta}$ are the radial and transverse components of the first Piola-Kirchhoff stress tensor $T$, and $\rho_{0}$ is the mass density of the matrix material in the reference configuration. The matrix material is considered to be an elastic/viscoplastic solid as the one considered by Tong and co-workers. ${ }^{19}$ The material is assumed to be homogenous, isotropic, and incompressible in plastic deformation, and its elastic moduli remains constant during plastic dcformation and under moderately high pressures. The rate form of constitutive relation for such a solid is (see Tong and co-workers ${ }^{19}$ with $\mathbf{W}=\mathbf{O}$ in this case):

$$
\dot{\mathbf{T}}-\left(\mathbf{D}-\mathbf{D}^{p}\right) \overline{\mathbf{T}}+\overline{\mathbf{T}}\left(\mathbf{D}^{p}-\mathbf{D}\right)=\mathbf{C}^{e}:\left(\mathbf{D}-\mathbf{D}^{p}\right),
$$

where $C^{e}$ is the elastic moduli tensor of the matrix material and takes the familiar form in Cartesian coordinates as

$$
C_{i j k l}^{e}=\lambda^{e} \delta_{i j} \delta_{k l}+\mu^{e}\left(\delta_{i k} \delta_{j l}+\delta_{i l} \delta_{j k}\right)
$$

where $\lambda^{e}$ and $\mu^{e}$ are the Lame constants.

The weighted Cauchy stress $\overline{\mathbf{T}}$ is defined as and the rate of plastic deformation tensor $\mathbf{D}^{p}$ is determined from the $J_{2}$ flow rule

$\mathbf{D}^{p}=\dot{\gamma} \frac{\mathbf{S}}{2 \tau_{e}}, \quad$ where $\mathbf{S}=\overline{\mathbf{T}}-\frac{1}{3} \operatorname{tr}(\overline{\mathbf{T}}) \mathbf{I}, \tau_{e}^{2}=\frac{1}{2} S_{i j} S_{i j}$,

where $\tau_{e}$ is the effective shear stress. The plastic shear strain rate $\dot{\gamma}$ is in general a function of applied stress, temperature, and internal state variables. ${ }^{28,29}$ The specific form of the plastic shear strain rate function used here is choscn to be a power law given by Klopp and co-workers, ${ }^{17}$

$$
\dot{\gamma}=\dot{\gamma}_{0}\left(\frac{\tau}{\tau_{r}}\right)^{1 / m}, \quad \tau_{r}=\tau_{0}\left(\frac{\gamma}{\gamma_{0}}\right)^{n}\left(\frac{\theta}{\theta_{0}}\right)^{\nu},
$$

where $\dot{\gamma}_{0}, \tau_{0}, \gamma_{0}$, and $\theta_{0}$ are reference strain rate, flow stress, plastic strain, and temperature, $\tau, \gamma$, and $\theta$ are current flow stress, plastic strain, and temperature, $m$ is the rate sensitivity, $n$ is the strain-hardening exponent, and $v$ is the thermal softening exponent.

\section{Spherical wave analysis}

For wave analysis it is advantageous to rewrite the rate form of elastic/viscoplastic constitutive relation given above in terms of the first Piola-Kirchhoff stress tensor $\mathbf{T}$ (noting only the diagonal components of $\mathbf{T}, \mathbf{F}, \mathbf{D}$, and $\mathbf{D}^{p}$ are nonzero):

$$
\dot{\mathbf{T}}=\mathbf{T D}+\mathbf{F}^{-1}\left(\mathbf{C}^{e}: \mathbf{D}\right)-\mathbf{2} \mathbf{T} \mathbf{D}^{p}-\mathbf{F}^{-1}\left(\mathbf{C}^{e}: \mathbf{D}^{p}\right),
$$

or, in the component form, as

$$
\begin{aligned}
& \frac{\partial T_{R}}{\partial t}=A_{1}(R, t) \frac{\partial u}{\partial R}-B_{1}(R, t), \\
& \frac{\partial T_{\theta}}{\partial t}=A_{2}(R, t) \frac{\partial u}{\partial R}-B_{2}(R, t),
\end{aligned}
$$

in which the coefficients $A_{1}, B_{1}, A_{2}$, and $B_{2}$ are

$$
\begin{aligned}
& A_{1}=\frac{r^{2}}{\eta R^{2}} T_{R}+\left(\lambda^{e}+2 \mu^{e}\right) \frac{r^{4}}{\eta^{2} R^{4}}, \\
& B_{1}=\left(2 T_{R}+\frac{2 r^{2}}{\eta R^{2}} \mu^{e}\right) D_{R}^{p}-\frac{2 r \lambda^{e}}{\eta R^{2}} u, \\
& A_{2}=(r / \eta R) \lambda^{e}, \\
& B_{2}=2\left(T_{\theta}+\frac{R}{r} \mu^{e}\right) D_{\theta}^{p}-\frac{T_{\theta}}{r} u-2\left(\lambda^{e}+\mu^{e}\right) \frac{R}{r^{2}} u .
\end{aligned}
$$

The rate form of constitutive equations combining with the balance equation of linear momentum, and the compatibility condition comprise a system of quasilinear, hyperbolic, partial differential equations for $T_{R}, T_{\theta}, u$, and $\eta$, 


$$
\begin{aligned}
& \frac{\partial T_{R}}{\partial t}=A_{1}(R, t) \frac{\partial u}{\partial R}-B_{1}(R, t), \\
& \frac{\partial T_{\theta}}{\partial t}=A_{2}(R, t) \frac{\partial u}{\partial R}-B_{2}(R, t), \\
& \frac{\partial T_{R}}{\partial R}+\frac{2}{R}\left(T_{R}-T_{\theta}\right)=\rho_{0} \frac{\partial u}{\partial t}, \\
& \frac{\partial \eta}{\partial t}=\frac{r^{2}}{R^{2}} \frac{\partial u}{\partial R}+\frac{2 \eta u}{r}
\end{aligned}
$$

This system can be written in terms of relations along characteristics

$$
\begin{aligned}
& d T_{R}= \pm \rho_{0} c d u-B_{1} d t \mp \frac{2}{R}\left(T_{R}-T_{\theta}\right) c d t \\
& \frac{d R}{d t}= \pm c= \pm\left(\frac{A_{1}}{\rho_{0}}\right)^{1 / 2}, \\
& d T_{\theta}=\frac{A_{2}}{A_{1}}\left(d T_{R}+B_{1} d t\right)-B_{2} d t, \quad \frac{d R}{d t}=0 \\
& d \eta=\frac{r^{2}}{R^{2} A_{1}}\left(d T_{R}+B_{1} d t\right)+\frac{2 \eta u}{r} d t, \quad \frac{d R}{d t}=0
\end{aligned}
$$

For infinitesimal deformations we have

$$
\eta \rightarrow 1, \quad r / R \rightarrow 1, \quad T_{R} / \mu^{e} \rightarrow 0, \quad \text { and } \quad T_{\theta} / \mu^{e} \rightarrow 0,
$$

so the above finite difference equations can be easily reduced to a simpler form (for example, see Hopkins ${ }^{30}$ ).

This system of characteristic relations Eq. (18) written in the form of a finite difference equations is solved by using a second-order accurate integration method, ${ }^{31}$ except, where large oscillations exist, a first-order accurate scheme is used. ${ }^{32}$ Because the material wave speed $c$ is strongly dependent on $r / R$, an updated nonuniform meshing scheme is adopted such that the accuracy of computation is maintained while the stability condition $c \Delta t / \Delta R$ $=\zeta \leqslant 1$ is satisfied for all nodes at each time step. For each time step, the current radius $r$ and the temperature $\theta$ are updated by

$$
d r=u d t \text { and } d \theta=\left(\beta \tau \dot{\gamma} / c_{p} \rho_{0}\right) d t,
$$

where the adiabatic condition is assumed for the spherical shell under dynamic loading by shock waves, and $c_{p}$ is the heat capacity of the matrix material and $\beta$ the fraction of the plastic work converted to heat. In general $\beta$ may depend on plastic strain and strain rate. ${ }^{33}$ In our present analysis we are interested in the consolidation of powders by shock wave propagation in which the rate of deformation of the bulk matrix material during pore collapse could be as high as $10^{7}$ per second. Due to the lack of experimental data on $\beta$ in the very high strain rate range $\left(10^{5}-10^{7}\right.$ per second ), $\beta$ is set to be a constant 0.9 in our calculations. In the following section, a detailed computational analysis of dynamic collapse of a viscoplastic spherical shell under external pressure is presented.

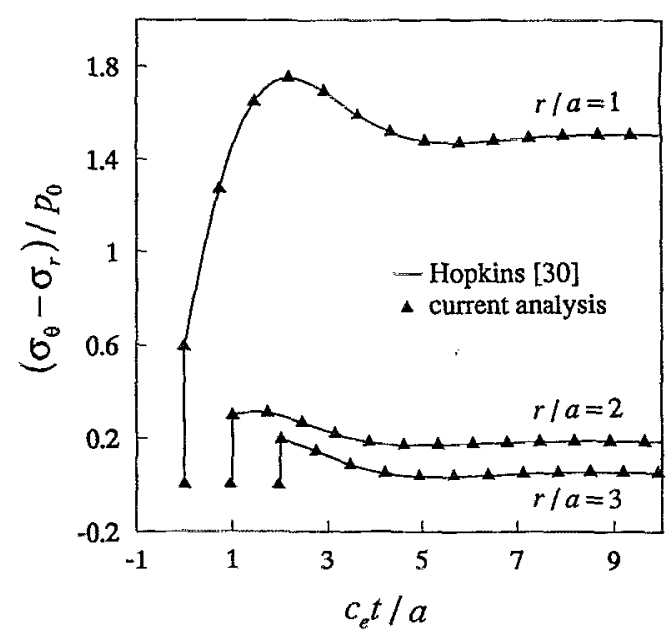

FIG. 1. Variation of shear stress $\left(\sigma_{\theta}-\sigma_{r}\right) / p_{0}$ with time $c_{e} t / a$ at various locations: $r / a=1$ (cavity surface), 2 , and 3. $p_{0}$ is the applied pressure, $c_{e}$ the elastic spherical wave speed, $a$ the cavity radius, and Poisson's ratio $v=0.29$.

\section{COMPUTATIONAL ANALYSIS AND RESULTS}

A numerical scheme for studying collapse of a spherical shell is implemented using the finite elastic/ viscoplastic deformation formulation described above. To verify the numerical scheme and to demonstrate the effectiveness of the current formulation presented here, several simple examples of spherical wave propagation are at first simulated and compared with those reported in the literature. The first example is the expansion of a cavity in an infinite elastic media when a pressure is applied suddenly on the interior surface of the cavity. The numerical result as well as the result from the analytical solution (see the review by Hopkins ${ }^{30}$ ) is given in Fig. 1. The numerical solution agrees with the analytical solution extremely well. The second example is the expansion of a cavity in an infinite elastic/perfectly plastic rate-independent media when a time-dependent pressure is applied on the interior surface of the cavity. ${ }^{34}$ In our formulation, we use a very small $m=0.005$ along with $n=v=0$ in our material model to represent a perfectly plastic, nearly rate-independent material. To avoid using a prohibitively small time step in our calculation, a maximum cutoff strain rate of $10^{9}$ per second is used. The numerical result is given in Fig. 2 along with the result by Friedman and co-workers. ${ }^{34}$ Once again, the agreement is excellent. Finally, we simulated the dynamic collapse of an aluminum spherical shell of elastic/ perfectly plastic rate-independent behavior when the outer surface of the spherical shell under a linearly increasing pressure loading and the inner surface of the shell is stress free. In particular, we simulated the case considered by Carroll and Holt ${ }^{16}$ as shown in their Fig. 5. Our numerical simulation gives essentially the same result (Fig. 3 ). The slight difference between the two results is due to the fact that we use $m=0.005$ (as opposed to $m=0$ for the rateindependent case) to approximate a rate-independent material. Thus, results of the dynamic analysis presented in 


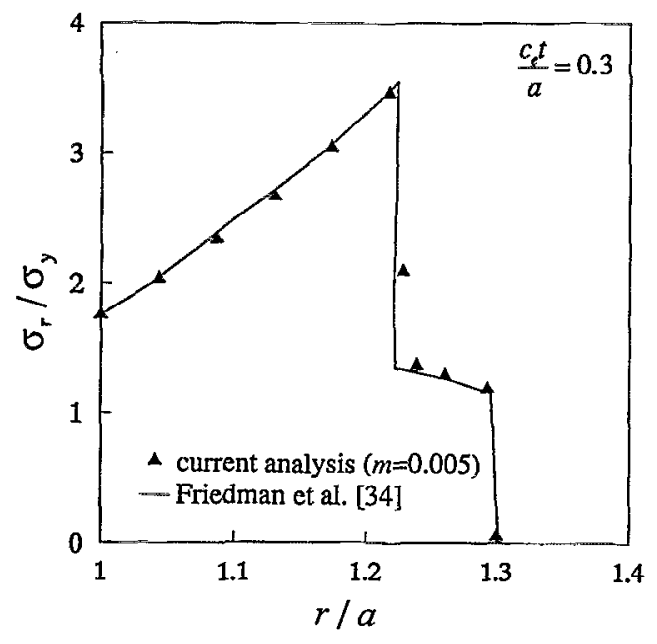

FIG. 2. Variation of radial stress $\sigma_{r} / \sigma_{y}$ with distance at the time $c_{e} t / a=0.3 . \sigma_{y}$ is the yield stress of the material, $a$ the cavity radius, $c_{e}$ the elastic spherical wave speed, the applied pressure on the cavity surface is $p(t)=-5 \sigma_{y} \exp \left(-3.5 c_{e} t / a\right)$, and Poisson's ratio $v=0.25$.

the following with $m=0.005$ for the matrix material can be regarded as a good approximation for a rate-independent material.

In the following we present the results of systematic parametric studies through a series of numerical simulation to illustrate the effect of various factors on the collapse of a spherical shell, including strain-rate sensitivity, inertia, and transient wave propagation, strain hardening, thermal softening, impulsive loading rate, pore size, and initial porosity. The matrix material is assumed to be pure aluminum in most of the calculations. We also include some of the results for pure iron and copper for comparison purposes. Relevant material parameters for these materials are listed in Tables I and II. Some computations of large problems have been carried out on a Cray supercomputer at

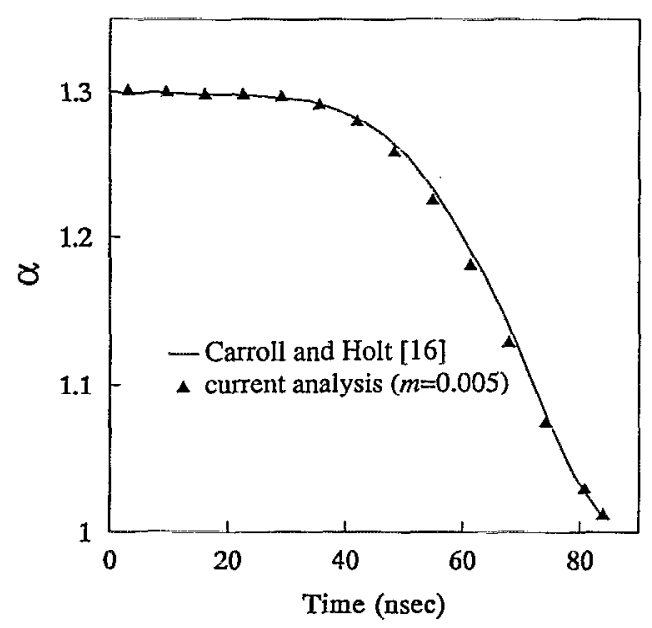

FIG. 3. Dynamic pore collapse of an aluminum hollow sphere of initial pore radius $a_{0}=20 \mu \mathrm{m}$ and initial relative density $\bar{\rho}_{0}=0.769$ (i.e. initial distention $\alpha_{0}=1.3$ ), with an applied external pressure rate $\dot{p}=10 \mathrm{MPa}$ / ns.
TABLE I. Selccted thermal-mechanical propertics of matrix matcrials.

\begin{tabular}{lccccr}
\hline Material & $\begin{array}{c}\text { Density } \\
\left(\mathrm{kg} / \mathrm{m}^{3}\right)\end{array}$ & $\begin{array}{c}\mu_{e} \\
(\mathrm{GPa})\end{array}$ & $\begin{array}{c}\lambda_{e} \\
(\mathrm{GPa})\end{array}$ & $\begin{array}{c}c_{p} \\
(\mathrm{~J} / \mathrm{kg} / \mathrm{K})\end{array}$ & $\begin{array}{c}\theta_{m} \\
(\mathbf{K})\end{array}$ \\
\hline Aluminum & 2700 & 26 & 56 & 893 & 920 \\
Iron & 7800 & 81 & 112 & 500 & 1530 \\
Copper & 8940 & 44 & 117 & 383 & 1356 \\
\hline \hline
\end{tabular}

San Diego Supercomputer Center and smaller problems are solved on an Ultrix DEC 3100 workstation. One of the important variables is the densification time at which the porous material is nearly fully densified. Because of the asymptotic nature of pore-collapse process as $\bar{\rho} \rightarrow 1$, the pore-collapse time or densification time $t_{c}$ is chosen to be the time when $\bar{\rho}_{c}=0.98$ for illustrative purposes.

Figure 4(a) shows the effects of strain rate sensitivity of the matrix materials and dynamic loading on the collapse process of a spherical shell under a linearly increasing loading with $\dot{p}=10 \mathrm{MPa} / \mathrm{ns}$. For simplicity as well as for direct comparison with the results of quasistatic analyses by Carroll and Holt ${ }^{16}$ for a rigid, perfectly plastic, rateindependent material and by Wilkinson and Ashby ${ }^{20}$ for a rigid, perfectly plastic, rate-dependent material under the same loading condition, effects of strain hardening and thermal softening are at first not included [i.e., $n=v=0$ in our model, Eq. (13)]. The result of the quasistatic analysis given by Carroll and Holt ${ }^{16}$ is

$$
\bar{\rho}=\left\{\begin{array}{cc}
\bar{\rho}_{0} \quad\left(0<p \leqslant p_{c}\right) \\
1-e^{-\sqrt{3} p / 2 \tau_{0}} & \left(p_{c} \leqslant p\right)
\end{array},\right.
$$

where $p_{c}=\left(2 \tau_{0} / \sqrt{3}\right) \ln \left[1 /\left(1-\bar{\rho}_{0}\right)\right]$,

and the result by Wilkinson and Ashby ${ }^{20}$ is

$$
\dot{\bar{\rho}}=\frac{\sqrt{3}}{2} \dot{\gamma}_{0} \frac{\bar{\rho}(1-\bar{\rho})}{\left[1-(1-\bar{\rho})^{m}\right]^{1 / m}}\left(\frac{\sqrt{3} m}{2} \frac{p}{\tau_{0}}\right)^{1 / m} \text {. }
$$

The time in Fig. $4(\mathrm{a})$ is normalized by $t_{0}$ which corresponds to the time when the applied pressure equals the yield stress in a perfectly plastic, rate-independent material, i.e., $p\left(t_{0}\right)=\sqrt{3} \tau_{0}$. The time $t_{0}$ can be regarded as a characteristic time of pore collapse for such a material in the quasistatic situation and hence is used as a reference time scale. Clearly in both quasistatic and dynamic analyses, a strong rate sensitivity retards the pore collapse. The pore collapse obtained from the present dynamic solution in all cases is faster than that predicted by static analysis initially but becomes slower at later times. Thus, the dynamic results by Carroll and $\mathrm{Holt}^{16}$ will give a much shorter densification time for rate-sensitive materials. Un-

\begin{tabular}{|c|c|c|c|c|c|c|c|}
\hline Material & $\left(\begin{array}{c}T_{0} \\
(\mathrm{MPa})\end{array}\right.$ & $\begin{array}{c}\dot{\gamma}_{0} \\
\left(10^{5} s^{-1}\right)\end{array}$ & $m$ & $\gamma_{0}$ & $n$ & $\begin{array}{c}\theta_{0} \\
(\mathrm{~K})\end{array}$ & $v$ \\
\hline Aluminum & 125 & 1.53 & 0.254 & 0.05 & 0.04 & 295 & -0.4 \\
\hline Iron & 420 & 4.0 & 0.20 & 0.15 & 0.085 & 295 & -0.6 \\
\hline Copper & 270 & 5.0 & 0.20 & $\ldots$ & $\ldots$ & $\cdots$ & . \\
\hline
\end{tabular}

TABLE II. Viscoplastic parameters for matrix materials. 

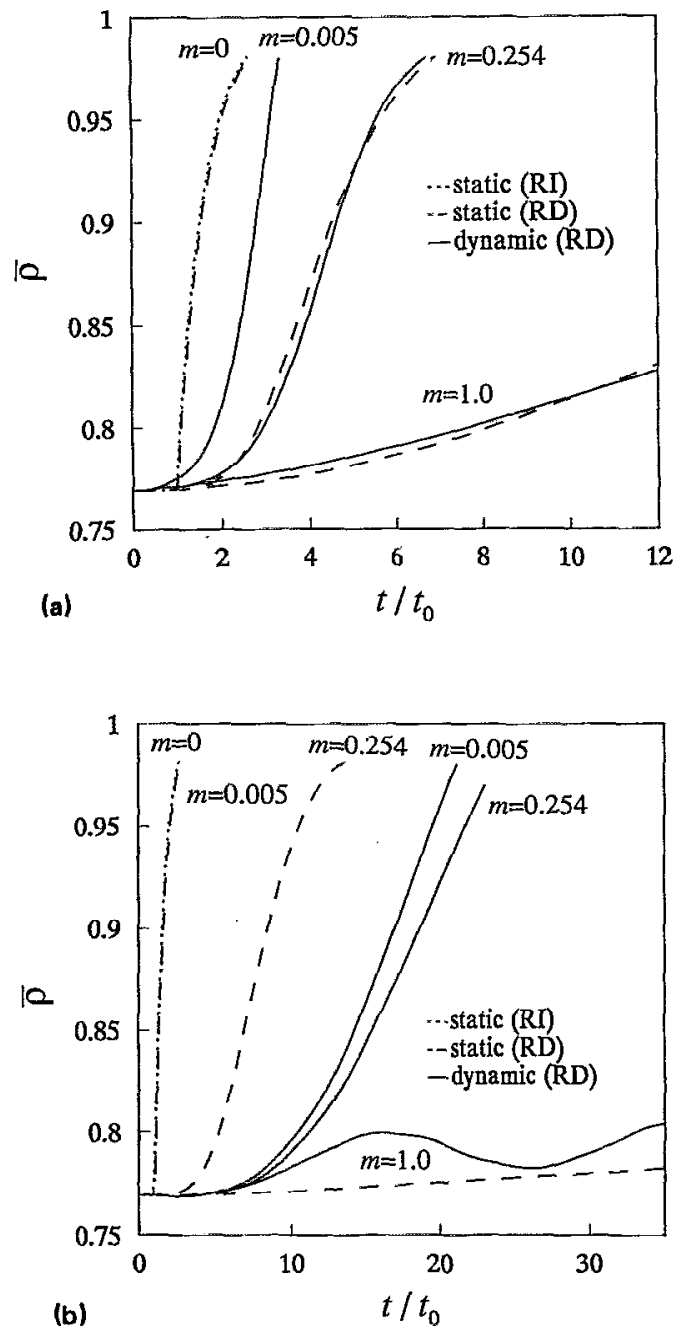

FIG. 4. Comparison of static and dynamic pore collapse of an elastic/ viscoplastic aluminum spherical shell of initial pore radius $a_{0}=20 \mu \mathrm{m}$ and initial relative density $\bar{\rho}_{0}=0.769$. The material model for plastic deformation in the current dynamic analysis is given as $\tau=\tau_{0}\left(\dot{\gamma} / \dot{\gamma}_{0}\right)^{m}:$ (a) With an applied external pressure rate $\dot{p}=10 \mathrm{MPa} / \mathrm{ns}, t_{0}=21.65 \mathrm{~ns}$; (b) with an applied external pressure rate $\dot{p}=250 \mathrm{MPa} / \mathrm{ns}$ and $t_{0}=0.866 \mathrm{~ns}$.

der this particular loading rate (relatively slow), the quasistatic and dynamic results are somewhat quite similar to each other when $m=0.254$ (for pure aluminum) or $m=1.0$ (the Newtonian viscous material). However, there is a large difference between static and dynamic analyses for $m=0.005$ (nearly rate independent) which indicates a strong dynamic effect in very weakly rate-sensitive materials even under relatively slow loading rate.

Variations of plastic strain rate, plastic shear strain, and temperature within the spherical shell are given in Figs. 5(a) $-5(\mathrm{c})$, respectively, for pure aluminum. The plastic strain rate is indeed of the order $10^{5}-10^{7}$ per second (note $\dot{\gamma}_{0}=1.53 \times 10^{5}$ per second). Initially the plastic strain rate is relatively uniform across the cross section. As the pore collapses, the plastic strain rate in the innermost region of the shell $\left(R / a_{0} \rightarrow 1\right)$ increases dramatically while the plastic strain rate reduces gradually in the outer region. The accumulated plastic shear strain also becomes nonuniform at the later stage of pore collapse, Fig. 5(b). Large- shear strain, as high as 2.86 , can be found near the inner surface of the shell at the time $t / t_{0}=4.78$ (at which $\bar{\rho}$ $=0.909$ ). At the same time, the temperature increases in that region due to adiabatic heating by plastic work to as high as $815 \mathrm{~K}$ ( $88 \%$ of the melting temperature of the matrix material) while the temperature at the outer surface of the shell remains relatively low (about $321 \mathrm{~K}$ ).

The influence of large plastic strain and temperature rise on dynamic pore collapse is examined by incorporating strain hardening and thermal softening into the viscoplastic model and the results are given in Fig. 6 for $m=0.254$. As expected, strain hardening slows down pore collapse (see the case with $n=0.25, v=0$ ) and thermal softening accelerates the process (see the case with $n=0, v=-1.0$ ). The result of dynamic pore collapse of pure aluminum using parameters given by Klopp and co-workers ${ }^{17}$ (see Table II) is found to be almost identical to the result using $m=0.254$ and $n=v=0$. The effect of strain hardening appears to annul the effect of thermal softening on dynamic pore collapse for pure aluminum. Because of this fact, only the simple viscoplastic model $(m=0.254, n=v=0)$ is used in the following calculations which can also be directly compared with the results of quasistatic analyses by Carroll and Holt ${ }^{16}$ and by Wilkinson and Ashby. ${ }^{20}$

So far, we have investigated the dynamic pore collapse of an aluminum spherical shell under a relatively low pressure rate of $\dot{p}=10 \mathrm{MPa} / \mathrm{ns}$. Figure $4(\mathrm{~b})$ presents the results using the same material parameters as in Fig. 4(a) but under a relatively high pressure rate of $\dot{p}=250 \mathrm{MPa} /$ ns. In contrast to the results in Fig. 4(a), there is large difference between quasistatic and dynamic analyses for all cases: $m=0.005,0.254$, and 1.0. The difference between the dynamic results with $m=0.005$ and $m=0.254$ in Fig. 4(b) is somewhat smaller than in Fig. 4 (a). For $m=1.0$, the dynamic analysis actually predicts a faster pore-collapse rate than that of static analysis for quite a while.

Figures 7(a) and 7(b) show the effect of pore size on dynamic pore collapse of the viscoplastic aluminum spherical shell $(m=0.254)$ under a low pressure rate of $\dot{p}=10$ $\mathrm{MPa} / \mathrm{ns}$ and a high pressure rate of $\dot{p}=250 \mathrm{MPa} / \mathrm{ns}$. For $\dot{p}=10 \mathrm{MPa} / \mathrm{ns}$, the results by dynamic analysis are very similar to those by static analysis for $a_{0}=2$ and $20 \mu \mathrm{m}$. For $a_{0}=100 \mu \mathrm{m}$ and larger, there is a strong dynamic effect even under a relatively slow pressure rate. For $a_{0}=50 \mu \mathrm{m}$, the dynamic pore collapse is evidently slower than static one but the densification time is shorter. For $\dot{p}=250 \mathrm{MPa} /$ $\mathrm{ns}$, the dynamic results for $a_{0}=20 \mu \mathrm{m}$ deviate largely from static ones. The dynamic effect becomes more dominant for larger pores of given initial relative density. The "critical" pore size under which both dynamic and static results are similar reduces as the applied pressure rate increases. There is no effect of pore size in quasistatic analysis. It is also interesting to note that the relative density of large pore sizes decreases by a large amount initially in our dynamic analysis as shown in Fig. 7(b), which is due to transient wave propagation and elastic deformation. Because the relative density is measured by $\bar{\rho}=1-(a / b)^{3}$, the rate of reduction in $b$ can be faster than that of $a$ during the initial collapse stage, thus it leads to a decrease in $\bar{\rho}$. 

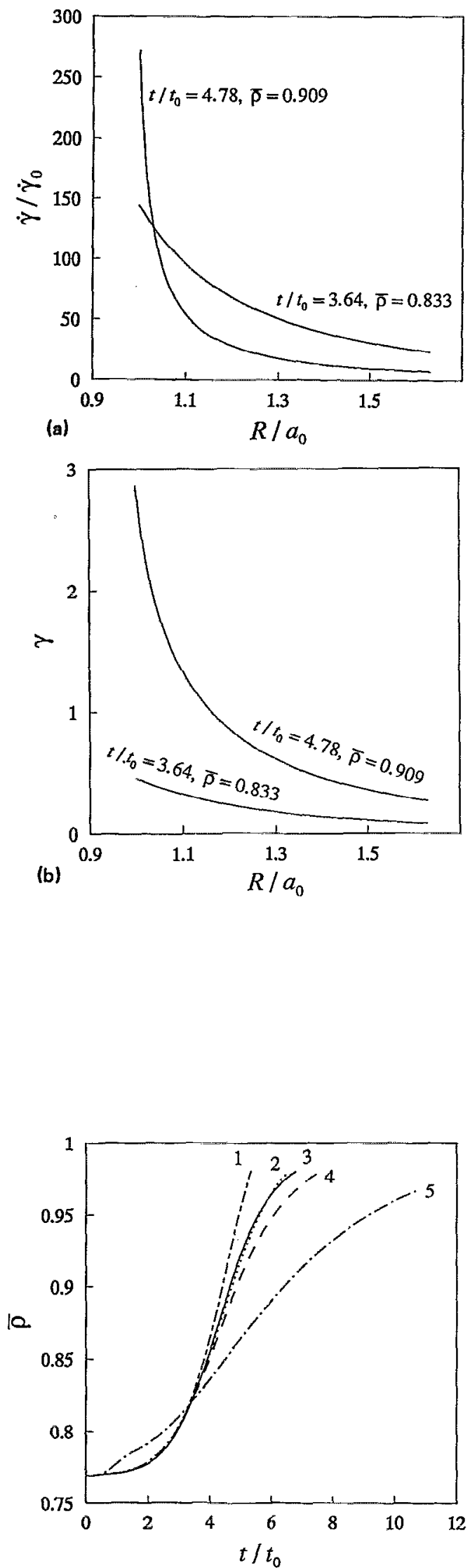

FIG. 6. Effect of strain hardening and thermal softening on dynamic pore collapse of an elastic/viscoplastic aluminum spherical shell: $a_{0}=20 \mu \mathrm{m}$, $\bar{\rho}_{0}=0.769, \dot{p}=10 \mathrm{MPa} / \mathrm{ns}, \tau=\tau_{0}\left(\dot{\gamma} / \dot{\gamma}_{0}\right)^{m}\left(\gamma / \gamma_{0}\right)^{n}\left(\theta / \theta_{0}\right)^{v}$, and $t_{0}=21.65$ ns: (1) $n=0, v=-1.0$; (2) $n=0.04, v=-0.4$; (3) $n=v=0$; (4) $n=0.04, v=0 ;(5) \quad n=0.25, v=0$.

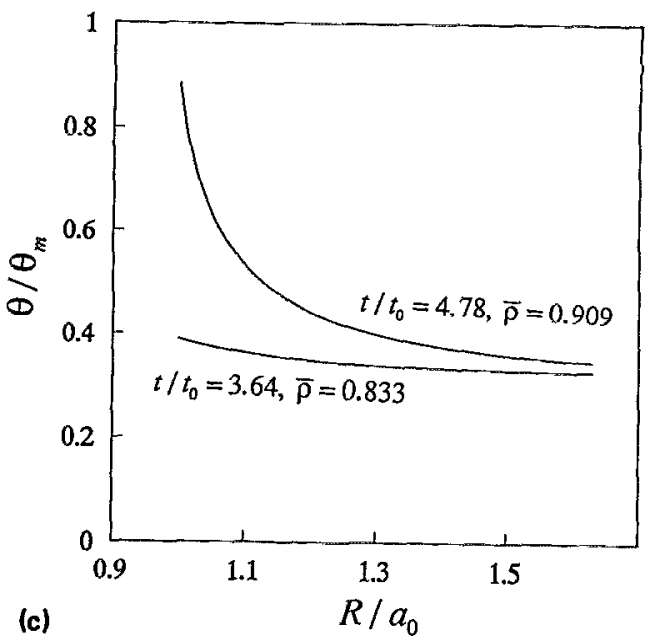

FIG. 5. Variations of (a) plastic shear strain rate, (b) plastic shear strain, and (c) temperature rise due to adiabatic heating of plastic work within the spherical shell at times $t / t_{0}=3.64(\bar{\rho}=0.833)$ and $t / t_{0}=4.78$ $(\bar{\rho}=0.909): a_{0}=20 \mu \mathrm{m}, \bar{\rho}_{0}=0.769, \dot{p}=10 \mathrm{MPa} / \mathrm{ns}, \tau=\tau_{0}\left(\dot{\gamma} / \dot{\gamma}_{0}\right)^{m}$, and $t_{0}=21.65 \mathrm{~ns}$. $R / a_{0}=1$ corresponds to the inner surface of the shell, and $R / a_{0}=1.603$ corresponds to the outer surface of the shell.

This transient phenomenon occurs in all dynamic analyses presented here and is more evident for larger pores and at higher loading rates.

The effcet of initial relative density on static and dynamic pore collapse of an aluminum spherical shell with the same pore size is also studied for both slow and fast pressure loading rates and results are given in Figs. 8(a) and $8(\mathrm{~b})$. The difference between static and dynamic analysis results is small for $\dot{p}=10 \mathrm{MPa} / \mathrm{ns}$ but becomes quite significant for $\dot{p}=250 \mathrm{MPa} / \mathrm{ns}$, with $\bar{\rho}_{0}=0.555,0.769$, and 0.909 . The retardation of the collapse process due to dynamic effect is evident for all three different initial relative densities. According to our dynamic analysis, the lower the initial relative density, the faster the pore collapses completely, which is in contrast to the results of quasistatic analysis.

In order to examine the effect of the strength and/or mass density-of-matrix materials on dynamic pore collapse, numerical simulations for pure aluminum, iron, and copper are carried out and results are presented in Fig. 9(a) under the same applied pressure history and in Fig. 9 (b) with the same static, rate-dependent pore-collapse response. The order of the densification times for aluminum, iron, and copper in dynamic analysis in terms of their respective static pore-collapse characteristic time is just opposite to that in static analysis, Fig. $9(\mathrm{a})$. This behavior 

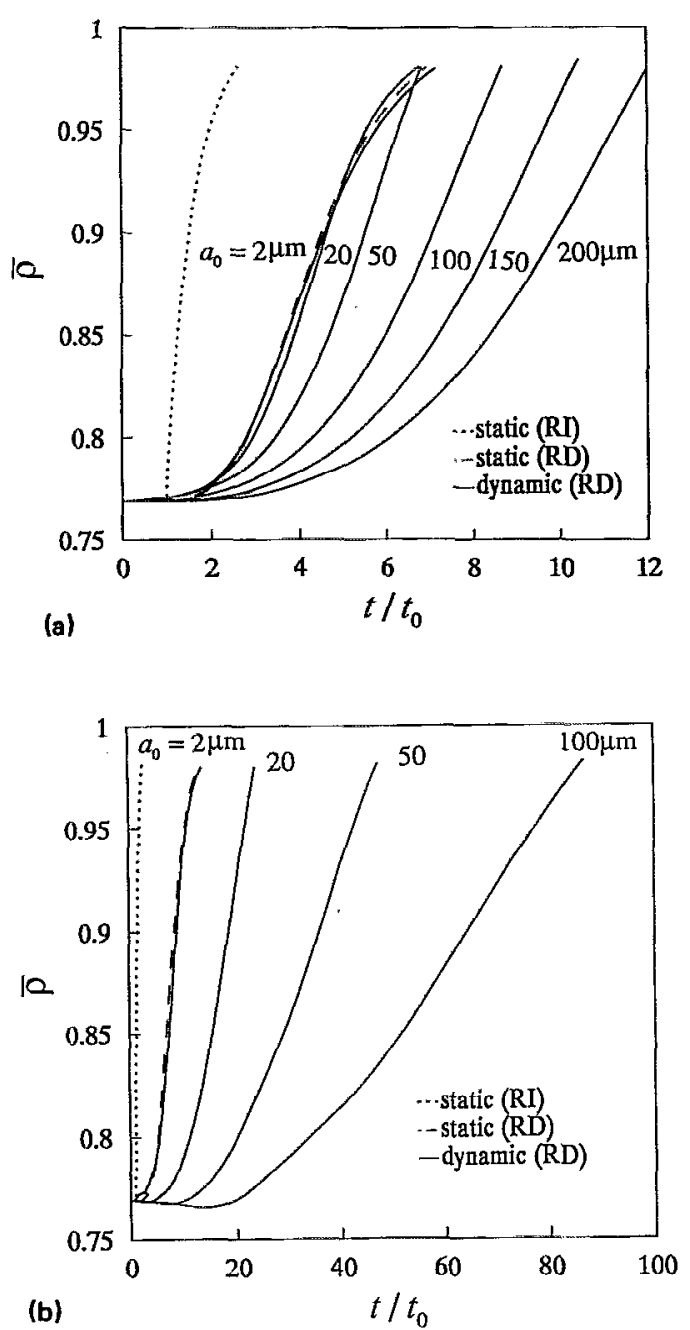

FIG. 7. Effect of pore size on static and dynamic pore collapse of an elastic/viscoplastic aluminum spherical shell: $\bar{\rho}_{0}=0.769, \tau=\tau_{0}\left(\dot{\gamma} / \dot{\gamma}_{0}\right)^{m}$, $\tau_{0}=125 \mathrm{MPa}, \dot{\gamma}_{0}=1.53 \times 10^{5} \ell / \mathrm{s}, m=0.254:$ (a) $a_{0}=2,20,50,100,150$, and $200 \mu \mathrm{m}$, with an applied external pressure rate $\dot{p}=10 \mathrm{MPa} / \mathrm{ns}$, $t_{0}=21.65 \mathrm{~ns}$; (b) $a_{0}=2,20,50$, and $100 \mu \mathrm{m}$, with an applied external pressure rate $\dot{p}=250 \mathrm{MPa} / \mathrm{ns}$ and $t_{0}=0.866 \mathrm{~ns}$.

should be expected because the dynamic effect is more dominant if the applied load is higher in comparison to the strength of the matrix material. In Fig. 9(b) the pressure rate is adjusted for each matrix material such that the quasistatic rate-dependent pore collapse is the same for all three matrix materials. The results from dynamic analysis are quite different from those of static analysis. The order of the densification times for aluminum, iron, and copper seems to correspond to the order of $\rho_{0} / \dot{p}$ for each matrix material, i.e., the higher the density of the matrix material, the larger the dynamic effect.

\section{CONCLUSIONS AND DISCUSSIONS}

The dynamic consolidation is accomplished by the passage of a shock wave through the powder. Major physical processes (rearrangement, deformation, and heating) occur during the densification process, i.e., during the shock rise time or within the shock front region. According to
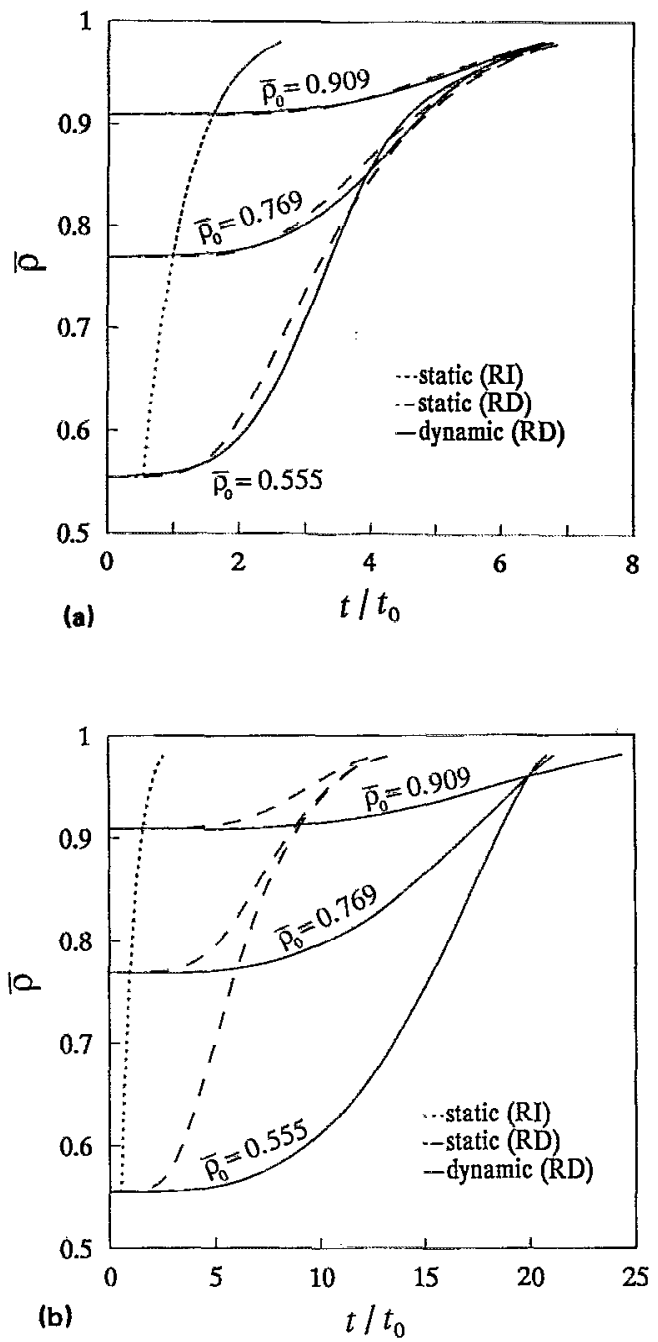

FIG. 8. Effect of initial relative density on static and dynamic pore collapse of an elastic/viscoplastic aluminum spherical shell: $a_{0}=20 \mu \mathrm{m}$, $\bar{\rho}_{0}=0.555,0.769$, and $0.909, \tau=\tau_{0}\left(\hat{\gamma} / \dot{\gamma}_{0}\right)^{m}$ : (a) with an applied external pressure rate $\dot{p}=10 \mathrm{MPa} / \mathrm{ns}, t_{0}=21.65 \mathrm{~ns}$; (b) with an applied external pressure rate $\dot{p}=250 \mathrm{MPa} / \mathrm{ns}$ and $t_{0}=0.866 \mathrm{~ns}$.

our computational analysis, the combined effects of inertia and strain-rate sensitivity strongly retard the densification of porous materials under impulsive loading, Figs. 4(a) and 4 (b). For a given loading rate, the viscous effect is more important when the matrix material becomes strongly rate sensitive $(m \rightarrow 1)$. The dynamic effect (inertia) is significant in weakly rate-sensitive matrix materials under all typical shock wave loading rates. For three typical elastic/viscoplastic materials studied here with $m \approx 0.2-0.25$, the difference in the densification time predicted by quasistatic and rate-independent analysis is very large. Even though the dynamic and rate-independent analysis reduces the difference to some degree its predicated densification time is still significantly smaller than that from the present dynamic and rate-dependent analysis, especially when the loading rate is moderately high. Under the condition given in Fig. 4(a), dynamic effects are small, thus the results by quasistatic and rate-dependent analysis are applicable to shock wave consolidation of alu- 

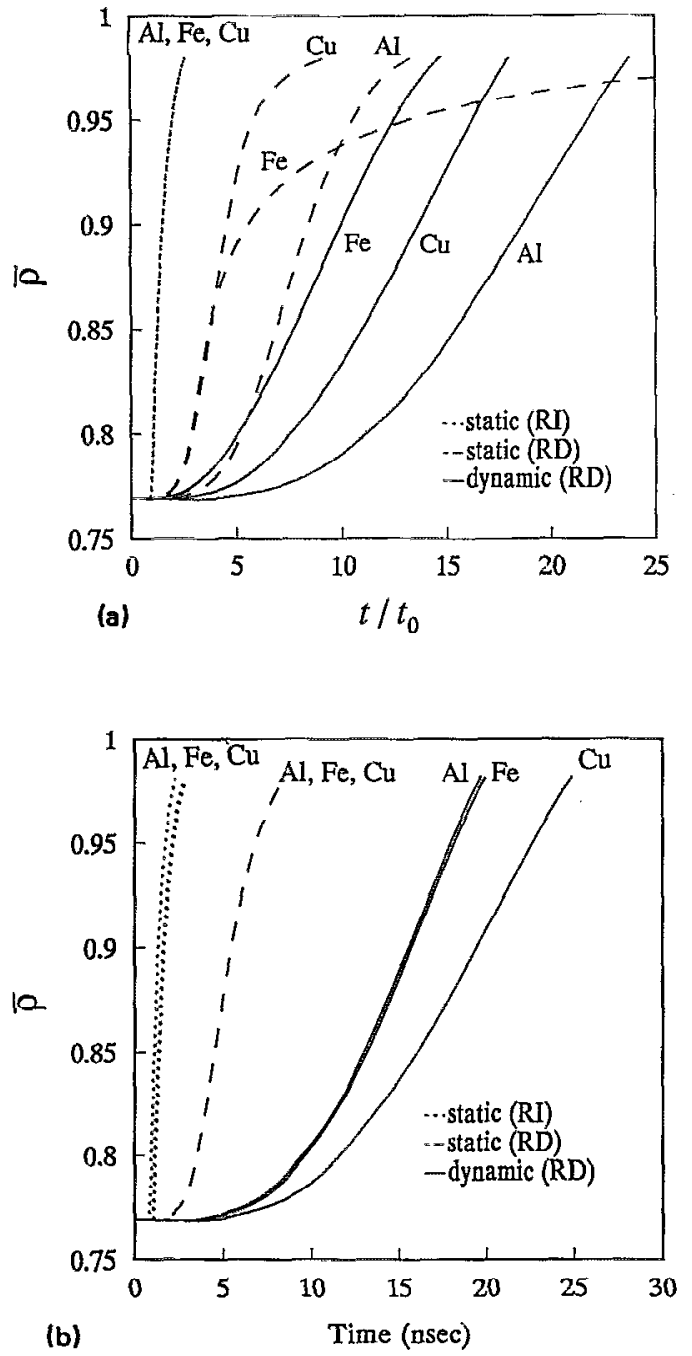

FIG. 9. (a) Comparison of static and dynamic pore collapse for aluminum, iron, and copper spherical shells with the same applied external pressure rate $\dot{p}=250 \mathrm{MPa} / \mathrm{ns}: a_{0}=20 \mu \mathrm{m}, \bar{\rho}_{0}=0.769, \tau=\tau_{0}\left(\dot{\gamma} / \dot{\gamma}_{0}\right)^{\mathrm{m}}$. Aluminum: $\tau_{0}=125 \mathrm{MPa}, \dot{\gamma}_{0}=1.53 \times 10^{5} \ell / \mathrm{s}, m=0.254, t_{0}=0.866 \mathrm{~ns}$; iron: $\tau_{0}=420 \mathrm{MPa}, \dot{\gamma}_{0}=4.0 \times 10^{5} \ell / \mathrm{s}, m=0.20, t_{0}=2.910 \mathrm{~ns}$; copper: $\tau_{0}=270$ $\mathrm{MPa}, \dot{\gamma}_{0}=5.0 \times 10^{5} \ell / \mathrm{s}, m=0.20, t_{0}=1.871 \mathrm{~ns}$. (b) Comparison of dynamic pore collapse for aluminum, iron, and copper spherical shells with the same static rate-dependent pore collapse: $a_{0}=20 \mu \mathrm{m}, \bar{\rho}_{0}=0.769$, $\tau=\tau_{0}\left(\dot{\gamma} / \dot{\gamma}_{0}\right)^{m}$. Aluminum: $\tau_{0}=125 \mathrm{MPa}, \dot{\gamma}_{0}=1.53 \times 10^{5} \ell / \mathrm{s}, m=0.20$, $\dot{p}=250 \mathrm{MPa} / \mathrm{ns}, \quad \rho_{0} / \dot{p}=10.8 \times 10^{3} \mathrm{~s} / \mathrm{m}^{2}$; iron: $\tau_{0}=420 \mathrm{MPa}$, $\dot{\gamma}_{0}=4.0 \times 10^{5} \mathrm{f} / \mathrm{s}, m=0.20, \dot{p}=693 \mathrm{MPa} / \mathrm{ns}, \rho_{0} / \dot{p}=11.3 \times 10^{3} \mathrm{~s} / \mathrm{m}^{2}$; copper: $\tau_{0}=270 \mathrm{MPa}, \dot{\gamma}_{0}=5.0 \times 10^{5} \ell / \mathrm{s}, \quad m=0.20, \dot{p}=426 \mathrm{MPa} / \mathrm{ns}$, $\rho_{0} / \dot{p}=20.9 \times 10^{3} \mathrm{~s} / \mathrm{m}^{2}$.

minum powders. Under a higher loading rate [Fig. 4(b)] or when the pore size is larger [Figs. 7(a) and 7(b)], dynamic effects become significant or even dominant. For Newtonian viscous fluid like solids $(m=1)$, dynamic effects are insignificant in most cases and results of quasistatic and rate-dependent analysis are applicable to dynamic consolidation process. The same conclusion has been reached for void growth in Newtonian viscous solids by Johnson. ${ }^{35}$

For simplicity, the pore-collapse time or densification time $t_{c}$ under a linearly increasing pressure loading (i.e., constant $\dot{p}$ ) can be written as

$$
t_{c}=\left(\sqrt{3} \tau_{0} / \dot{p}\right) g\left(\dot{p}, \tau, \rho_{0}, a_{0}, \bar{\rho}_{0}\right)
$$

The pore-collapse time to a good approximation can be regarded to be independent of initial porosity or initial relative density [see Figs. 8(a) and $8(\mathrm{~b})$ ]. For quasistatic loading and rate-independent matrix materials, $g \approx 1$. When dynamic effects are insignificant (for example, $m \approx 1$, or a small pore size or a low pressure rate), $g \approx g_{R}$ for rate-dependent matrix material, where $g_{R}$ is a normalized quasistatic and rate-dependent pore-collapse time and can be computed by using the results for relative density rate from quasistatic analysis of rate-dependent, strainhardening, and thermal softening matrix materials. For power-law perfectly viscoplastic materials (Wilkinson and Ashby $\left.{ }^{20}\right), g_{R}$ can be obtained as [see Eq. (22)]

$$
g_{R}=\left(\frac{G}{F}\right)^{m /(1+m)}
$$

where

$$
\begin{aligned}
& G=\int_{\bar{\rho}_{0}}^{\dot{\rho}_{c}} \frac{\left[1-(1-\bar{\rho})^{m}\right]^{1 / m}}{\bar{\rho}(1-\bar{\rho})} d \bar{\rho}, \\
& F=\frac{m}{1+m}\left(\frac{3 m}{2}\right)^{(1+m) / m} \frac{\tau_{0} \dot{\gamma}_{0}}{\dot{p}} .
\end{aligned}
$$

For strain-hardening viscoplastic materials, the results such as the one by Haghi and Anand ${ }^{2 S}$ may be used to evaluate $g_{R}$. Finally, when dynamic effects are not negligible (the pressure loading rate is high for a given matrix material and porc sizc), $g \approx g_{R} g_{D}$, wherc $g_{D}$ is a nondimensional parameter, a measure of dynamic effects $(\geqslant 1)$. The dynamic parameter $g_{D}$ may be in general dependent on pressure loading rate, viscoplastic properties of matrix materials, and initial pore size. For a given matrix material, $g_{D}$ may be tabulated for different loading rates or different pore sizes. Furthermore, $g_{D}$ can also be used as a criterion in determining whether the pressure loading rate is low or high for a given matrix material and pore size: The pressure loading rate is low when $g_{D} \approx 1$ and the pressure loading rate is high when $g_{D} \gg 1$.

Schwarz and co-workers ${ }^{14}$ reported measurements on the temperature rise time of about 60-70 ns during shock wave consolidation of copper-constantan powders $(60 \mu \mathrm{m}$ in diameter) for shock pressures between 1.3 and $9.4 \mathrm{GPa}$. The maximum temperature can be regarded to be due to the shock wave heating as the powder is being fully densified and hence the temperature rise time is a good measure of the densification time by shock wave. The relatively large rise time is in itself an indicator of dynamic and strain-rate effects on consolidation process. The finite rise time is commonly attributed to the time for the shock wave passing through 1-2 or 3-5 powder particles. ${ }^{2,15}$ However, the temperature rise times measured for two tests no. 801 and no. 802 are 67 and 63 ns, respectively while the shock velocities in copper powder for these two tests are 0.75 and $2.03 \mathrm{~km} / \mathrm{s}^{14}$ The times for these two shock waves passing through 1.5 particles $(90 \mu \mathrm{m})$ are about 120 and $44 \mathrm{~ns}$, respectively. Such a big difference in rise time is not observed in the experimental measurements, rather, the measured temperature rise times are almost identical. The ex- 
perimental observation could be partly explained in terms of the dynamic effects on pore collapse. The shock pressures in these two experiments are 1.3 and $9.4 \mathrm{GPa}$, respectively, and thus the pressure loading rates $\dot{p}$ are 19.4 and $149.2 \mathrm{MPa} / \mathrm{ns}$, respectively. The dynamic effects will be stronger in the later test (higher-pressure loading rate) and thus retard the densification process.

As mentioned in Sec. I, constitutive relations of porous materials, especially such as the one proposed by Herrmann, ${ }^{11}$ are commonly used in computer simulation of dynamic consolidation by shock wave propagation. The crush curve (i.e., the $p-\alpha$ relation), however, is often taken to be that determined from quasistatic tests and without the inclusion of rate effects..$^{11,15,36}$ Acceptable for certain rate-insensitive materials under slow (low rate) loading conditions, ${ }^{37}$ the quasistatic rate-independent $p-\alpha$ relation cannot be assumed to be valid in general applications of shock wave consolidation without careful examination of effects of inertia and strain rate. If the quasistatic rateindependent $p-\alpha$ relation is used in modeling shock consolidation, the finite rise time of the shock wave (the finite width of the shock front) can only be artificially imposed (say, by numerical viscosity). On the other hand, from our present analysis, it can be seen that the finite rise time is the natural consequence of a rate-dependent and dynamic pore-collapse process during the passage of the consolidation shock wave. Thus, a rate-dependent and dynamic $p-\alpha$ relation will improve the modeling of physical processes during shock wave consolidation. As pointed out by Carroll and Holt, ${ }^{16}$ even for perfectly plastic and rateindependent materials, a dynamic pore-collapse relation becomes extremely complex (a second-order nonlinear ordinary differential equation). However, approximate dynamic $p$ - $\alpha$ relations may be obtained for somc simple loading cases. As a first approximation, quasistatic but ratedependent pore-collapse relations such as the one proposed by Wilkinson and Ashby ${ }^{20}$ [Eq. (22)] should be used for $m \approx 1$, or a small pore size, or a low pressure rate (i.e., when $g_{D} \approx 1$ ). For a given loading condition in which dynamic effects are not negligible $\left(g_{D}>1\right)$, a modified quasistatic and rate-dependent relation may be used instead. For example, Eq. (22) may be modified as

$$
\dot{\rho}=D^{*} \frac{\sqrt{3}}{2} \dot{\gamma}_{0} \frac{\bar{\rho}(1-\bar{\rho})}{\left[1-(1-\bar{\rho})^{m}\right]^{1 / m}}\left(\frac{\sqrt{3} m}{2} \frac{p}{\tau_{0}}\right)^{1 / m},
$$

where $D^{*}(<1)$ is a nondimensional coefficient to account for dynamic effects. Again, for a given matrix material, $D^{*}$ can be tabulated for different loading rates or different pore sizes.

The localized melting near particle surfaces is another important aspect of shock consolidation. Large temperature rises in pore interior surface are predicted by our dynamic pore collapse analysis [Fig. 5(c)]. Heat conduction is neglected here (although it can easily be incorporated into our current analysis) because the times required for densification process are very short $(\approx 100 \mathrm{~ns})$. For some low-pressure loading rate cases or if the matrix material is very strongly rate sensitive (say, a Newtonian viscous solid with $m=1$ ), the time for complete consolidation of pow- ders may be longer ( $1 \mu$ s or more) and an adiabatic condition will be no longer valid. While large plastic deformation, frictional sliding, and other processes contribute to the localized heating, frictional sliding between particles has commonly been regarded to be the dominant factor, ${ }^{9,38}$ however, the conclusion may not be completely justified. The estimate of frictional work using a Coulomb friction model (constant coefficient of friction) is questionable for very high-pressure contact between particles encountered in shock wave loading. ${ }^{39-41}$ The sliding friction stress is essentially limited by the shear strength of matrix material under high pressure. ${ }^{39,40}$ For closely or near closely packed powders, the relative sliding distance between particles is very small (of the order of several micrometers or less). Due to surface oxide layer and other contaminations, the sliding friction between dry surfaces is also strongly dependent on the sliding distance such that the friction stress during the initial sliding stage is relatively much smaller than that in the steady-state stage. ${ }^{40,41}$ Interparticle surfaces will soften and melt due to the heating by frictional sliding and will not transmit any significant friction stress afterward. Thus, the frictional working would be much smaller than that estimated by simple dry Coulomb friction law.

The plastic work of the viscoplastic matrix material during consolidation may be underestimated by assuming homogenous deformation of perfectly plastic and rateindependent material. The deformation of a spherical shell under pressure loading [Fig. 5(b)] is quite nonuniform and the plastic work done upon the pore collapse of porous materials can be quite large. Finally, a recent experimental study and analysis by Mutz ${ }^{42}$ on the fraction of the melt after shock wave consolidation concludes that the assumption of all heat flowing into the particle surface (such as by frictional sliding) gives an overestimate of the melt fraction and even predicts the possibility of vaporization of the particle surface layer which is unrealistic. $\mathrm{He}$ found that a $70 \%$ bulk and $30 \%$ surface layer partition of heat flow from shock energy input (which is similar to viscoplastic pore collapse) provides the best agreement with the experimental data. From available experimental results we conclude that the present dynamic pore-collapse analysis describes not only the densification process but also to a significant degree the surface melting of particles through adiabatic heating due to plastic work.

One of the implications of our computational results is that the dynamic pore collapse of a spherical shell can be used to study material response under very large strain and strain rates. In particular, because of the strong strain-rate and dynamic effects for matrix materials with large $m$ (say, $0.2-1$, the combined experimental and computational investigation can be carried out to evaluate such as the rate sensitivity of materials at very high strain rates. This may also provide a sensitive method to rigorously examine the hypothesis whether the behavior of Newtonian viscous fluids $(m=1)$ prevails at strain rates higher than $10^{4}$ per second.

While most efforts have been directed toward the understanding of the consolidation process by shock wave, ${ }^{2,4}$ 
much less attention has been paid to problems of cracking in near fully densified compacts due to release tensile waves. Our current analysis can be easily extended to more complicated loading paths such as compressive-tensile loading and to include more complex viscoplastic models, especially those with strain-rate history effects. ${ }^{19,43}$ The effect of dynamic loading and viscoplastic properties of matrix materials on void growth (and eventually cracking) in compacts can be assessed. Furthermore, there is an increasing application of continuum, phenomenological constitutive relations of porous material to dynamic ductile fracture, ${ }^{44}$ and spallation. ${ }^{45}$ The flow potentials of porous materials used in these analyses are based on quasistatic results. The correction due to dynamic effects on void growth using those flow potentials can also be examined using our analysis.

\section{ACKNOWLEDGMENTS}

The authors would like to acknowledge the support of this research by the Division of Materials Research of the National Science Foundation through Grant No. DMR9116570. G.R. acknowledges the support of a NSF Presidential Young Investigator award, Grant No. MSS9157846. Discussions with Professor T. Vreeland, Jr. and Professor T. Christman on shock wave consolidation are gratefully acknowledged. The computations were performed on a Cray Y-MP at the San Diego Supercomputer Center (SDSC).

${ }^{1}$ V. D. Linse, Dynamic Compaction of Metal and Ceramic Powders, NMAB-394, (National Academy Press, Washington, DC, 1983).

${ }^{2}$ R. B. Schwarz, P. Kasiraj, T. Vreeland, Jr., and T. J. Ahrens, Acta Metall. 32, 1243 (1984).

${ }^{3}$ W. H. Gourdin, Prog. Mater. Sci. 30, 39 (1986).

${ }^{4}$ Y. Horie, R. A. Graham, and I. K. Simonsen, Mater. Lett. 3, 354 (1985).

${ }^{5}$ N. N. Thadhani, Adv. Mater. Manufac. Proc. 3, 493 (1988).

${ }^{\circ} T$. Christman, K. Heady, and T. Vreeland, Jr., Scr. Metall. 25, 631 (1991).

${ }^{7}$ R. A. Berry and R. L. Williamson, in Shock Waves in Condensed Matter, edited by Y. M. Gupta (Plenum, New York, 1986), p. 337.

${ }^{3}$ J. E. Flinn, R. L. Williamson, R. A. Berry, R. N. Wright, Y. M. Gupta, and M. Williams, J. Appl. Phys. 64, 1446 (1988)

${ }^{9}$ J. Gao, B. Shao, and K. Zhang, J. Appl. Phys. 69, 7547 (1991).

${ }^{10} \mathrm{~A}$. Ferreira and M. A. Meyers, in Shock-Wave and High-Strain-Rate Phenomena in Materials, editcd by M. A. Mcycrs, L. E. Murr, and K. P. Staudhammer (Marcel Dekker, New York, 1992), p. 361.

"W. Herrmann, J. Appl. Phys. 40, 2490 (1969).

${ }^{12}$ M. M. Carroll and A. C. Holt, J. Appl. Phys. 43, 759 (1972).

${ }^{13}$ J. W. Swegle, J. Appl. Phys. 51, 2574 (1980).
${ }^{14}$ R. B. Schwarz, P. Kasiraj, and T. Vreeland, Jr., in Metallurgical Applications of Shock Waves and High-Strain-Rate Phenomena, edited by L. E. Murr, M. A. Meyers, and K. P. Staudhammer (Marcel Dekker, New York, 1986), p. 313.

${ }^{15}$ D. Raybould and T. Z. Blazynski, in Materials at High Strain Rates, edited by T. Z. Blazynski (Elsevier, New York, 1987), p. 71.

${ }^{16}$ M. M. Carroll and A. C. Holt, J. Appl. Phys. 43, 1626 (1972).

${ }^{17}$ R. W. Klopp, R. J. Clifton, and T. G. Shawki, Mech. Mater. 4, 375 (1985).

${ }^{18}$ R. J. Clifton, Appl. Mech. Rev., 43, s9 (1990).

${ }^{19}$ W. Tong, R. J. Clifton, and S. Huang, J. Mech. Phys. Solids 40, 1251 (1992).

${ }^{20}$ D. S. Wilkinson and M. F. Ashby, Acta Metall. 23, 1277 (1975).

${ }^{21}$ B. Budiansky, J. W. Hutchinson, and S. Slutsky, in Mechanics of Solids, The Rodney Hill 60th Anniversary Volume, edited by H. G. Hopkins and M. J. Sewell (Pergamon, New York, 1982), p. 13.

${ }^{22}$ M. M. Carroll, Metall. Trans. A 17, 1977 (1986).

${ }^{23}$ K. T. Kim and M. M. Carroll, Int. J. Plasticity 3, 67 (1987).

${ }^{24}$ M. Hori and S. Nemat-Nasser, J. Appl. Phys. 64, 856 (1988).

${ }^{25} \mathrm{M}$. Haghi and L. Anand, Int. J. Plasticity 7, 123 (1991).

${ }^{26}$ L. Seaman, R. E. Tokheim, and D. R. Curran, Stanford Research Institute Report PYU-2407, 1974.

${ }^{27} \mathrm{~L}$. E. Malvern, Introduction to the Mechanics of a Cantinuous Medium (Prentice-Hall, New Jersey, 1969), p. 670.

${ }^{28}$ J. R. Rice, J. Appl. Mech. Trans. ASME 37, 728 (1970).

${ }^{29}$ R. J. Clifton, J. Appl. Mech. Trans. ASME 50, 941 (1983).

${ }^{30}$ H. G. Hopkins, in Progress in Solid Mechanics, edited by I. N. Sneddon and R. Hill (Wiley-Interscience, New York, 1960), Vol. 1, p. 85.

${ }^{31} \mathrm{~S}$. Ranganath and R. J. Clifton, Comp. Meth. Mech. Eng. 1, 173 (1972).

${ }^{32}$ W. Tong, Ph.D. thesis, Brown University, Providence, RI, 1991.

${ }^{33}$ J. J. Mason, A. J. Rosakis, and G. Ravichandran, to appear in Mechanics of Materials: Special Volume on Shear Instabilities and Viscoplasticity Theories Dedicated to the Memory of J. Duffy, edited by R. W. Armstrong, R. C. Batra, M. A. Meyers, and T. W. Wright (Press, City, in press).

${ }^{34}$ M. B. Friedman, H. H. Bleich, and R. J. Parnes, J. Eng. Mech. Proc. ASCE EM3, 189 (1965).

${ }^{35}$ J. N. Johnson, J. Appl. Phys. 52, 2812 (1981).

${ }^{36}$ R. K. Linde, L. Seaman, and D. N. Schmidt, J. Appl. Phys. 43, 3367 (1972).

${ }^{37}$ M. da Silva and K. T. Ramesh, J. Phys. IV Colloq. 1, C3-909 (1991).

${ }^{38}$ T. J. Ahrens, G. M. Bond, W. Yang, and G. Liu, in Shock-Wave and High-Strain-Rate Phenomena in Materials, edited by M. A. Meyers, L. E. Murr, and K. P. Staudhammer (Marcel Dekker, New York, 1992), p. 339.

${ }^{39}$ W. Tong, L. Anand, D. M. Parks, A. C. Pisoni, and L. Cheng (unpublished).

${ }^{40} \mathrm{~T}$. Wanheim, Wear 25, 225 (1973).

${ }^{41} \mathrm{~V}$. Prakash and R. J. Clifton, in Proceedings of the VII International Congress on Experimental Mechanics (Society for Experimental Mechanics, Bethel, CT, 1992), p. 556.

${ }^{42}$ A. Mutz, Ph.D. thesis, California Institute of Technology, Pasadena, CA, 1992.

${ }^{43}$ P. S. Follansbee and U. F. Kocks, Acta Metall. 36, 81 (1988).

${ }^{44}$ A. Needleman and V. Tvergaard, InL. J. Fract. 49, 41 (1991).

${ }^{45}$ J. Eftis and J. A. Nemes, Int. J. Plasticity 7, 275 (1991). 